\title{
Structural organization of sterol molecules in DPPC bilayers: A Coarse-Grained Molecular Dynamics investigation ${ }^{\dagger}$
}

\author{
Yawen Zhang $^{a \dagger}$, James Carter $^{a \dagger}$, Anders Lervik ${ }^{a \ddagger}$, Nicholas J. Brooks ${ }^{a}$, John M. Seddon ${ }^{a}$ and Fer- \\ nando Bresme* ${ }^{* a}$
}

\author{
Received Xth $X X X X X X X X X X 20 X X$, Accepted Xth $X X X X X X X X X 20 X X$ \\ First published on the web Xth $X X X X X X X X X X 200 X$ \\ DOI: $10.1039 / b 000000 x$
}

We investigate the structural organization of cholesterol (CHOL) analogues in 1,2-dipalmitoyl-sn-glycero-3-phosphocholine (DPPC) bilayers using coarse-grained molecular dynamics simulations and the MARTINI forcefield. Different sterol molecules are modelled by increasing (CHOLL) or decreasing (CHOLS) the diameter of the sterol beads employed in the MARTINI model of CHOL. At high sterol concentrations, $\left(x_{\text {sterol }}=0.5\right)$, typical of liquid ordered phases, we find that the sterol arrangement and sterol-DPPC interactions strongly depend on the sterol size. Smaller sterols (CHOLS and CHOL) form linear clusters, while the larger sterols (CHOLL) arrange themselves into disc shaped clusters. By combining structural and dynamical properties we also investigate the $\mathrm{S}_{\mathrm{o}} \rightarrow \mathrm{L}_{\mathrm{d}}$ transition for the CHOLL and CHOLS sterols. We show that small changes in the sterol size significantly affect the stability of the gel phase with the gel phase stabilized by the small sterols, but destabilized by large sterols. The general dependence of the phase behaviour of the membrane with sterol content, is reminiscent of the one observed in naturally occurring membranes. The relevance of our results to understand current cholesterol-bilayer structural models is discussed.

\section{Introduction}

Cholesterol (CHOL) is one of the most important molecules in mammalian cell membranes and an essential structural component that regulates the membrane permeability and fluidity. ${ }^{1}$ It is well established that CHOL affects the membrane phase behaviour. It induces the so called condensing effect,, 213 and modifies the membrane thickness, $\frac{4}{4}$ as well as the membrane mechanical properties. ${ }^{[5}$ The importance of cholesterol in promoting the formation of small lipid "rafts", which are considered to mediate signal transduction, has also been highlighted. ${ }^{6}-8$ Further, Ipsen et al., predicted the formation of the "liquid-ordered" $\left(\mathrm{L}_{\mathrm{o}}\right)$ phase ${ }^{9}$ at high cholesterol content. This phase has been corroborated in a number of experimental and simulation studies. 140

The structure of cholesterol in biological membranes has been studied for a number of years. ${ }^{15 / 16}$ Three different models have been developed to rationalize the sterol-lipid interactions. The Condensed Complex Model was proposed following experimental observations in lipid monolayers adsorbed at the vapor-water interface. ${ }^{17}$ This model postulates the formation of sterol-phospholipid complexes, an idea that was prompted by the observation of a liquid-liquid critical

${ }^{a}$ Department of Chemistry, Imperial College London, United Kingdom. Email: f.bresme@imperial.ac.uk

$\ddagger$ Present Address: Department of Chemistry, Norwegian University of Science and Technology, Trondheim, Norway.

$\dagger$ These authors contributed equally to this work. point in mixtures containing dihydrocholesterol and phospholipids. The Superlattice Model assumes that the CHOL molecules embedded in the fluid membrane are arranged in hexagonal superlattices, with a CHOL-CHOL separation distance $\sim 2-3$ times the effective diameter of the phospholipid molecules. $\frac{1819}{19}$ Finally, the Umbrella Model takes into account the distinct molecular structure of cholesterol, consisting of a small hydrophilic head group and a bulky rigid hydrophobic tetrameric ring. According to the umbrella model, the size mismatch between the head and tail regions of the CHOL and phospholipid molecules favours the aggregation of phospholipids around the CHOL, hence allowing the larger head group of the phospholipids to help shield the CHOL molecules from the water phase. In this way, unfavourable interactions between water and the non-polar region of cholesterol can be minimised. This model predicts that CHOL molecules have a higher tendency to disperse in the bilayer rather than cluster together. 20

Computer simulations can provide important structural information to test the validity of the CHOL-phospholipid models. Earlier atomistic simulations $21-23$ have shown that the two off-plane methyl groups in CHOL significantly influence the CHOL lateral structure in the bilayer. When the offplane methyl groups are removed, hence rendering a "flatter" molecule, the sterols arrange into linear clusters, while the original CHOL molecules form triangular clusters. These simulations highlight the strong sensitivity of the sterol-sterol and sterol-phospholipid interactions to small changes in the 
sterol structure. We note that the CHOL molecule has a crosssectional area of $0.27 \mathrm{~nm}^{2}, 24$ which lies in between that of a single saturated-acyl chain $\left(0.21 \mathrm{~nm}^{2}\right)^{24}$ and that of two acyl chains $\left(0.42 \mathrm{~nm}^{2}\right)^{24}$ when the phospholipids are in the gel phase. Thus, addition of CHOL to the membrane results in a strong mismatch of the phospholipid packing, and consequently a significant disruption in the 2D phospholipid hexagonal lattice. Hence a strong sensitivity of the membrane properties to the effective size of the sterol is expected. This hypothesis motivates the present work. We expect that modifications of the sterol size that result in sterol areas closer to the characteristic acyl chain area, should result in a better stabilisation of the gel phase upon addition of sterols. There is indeed evidence that the use of appropriate additives, e.g. alcohols, can stabilize the gel phase. As a matter of fact, tetradecanol ( 0.8 mol fraction) can increase the melting temperature of the DMPC bilayer from $23.5^{\circ} \mathrm{C}$ to $58.9^{\circ} \mathrm{C} .25$

In order to understand the role of the sterol size in the phase behaviour of biological membranes and on the clustering of the sterol molecules in the membrane, we have performed a systematic analysis of sterol analogs by using the coarsegrained (CG) MARTINI forcefield. 26 We have shown in our previous work that the addition of cholesterol induces the gel$\mathrm{L}_{\mathrm{o}}$ phase transitions in DPPC bilayers, with the $\mathrm{L}_{\mathrm{o}}$ phase being stable at high CHOL molar fractions, $\left(x_{\text {sterol }} \sim 0.5\right)$, over a wide range of temperatures. ${ }^{14}$ Interestingly, we found evidence for the formation of linear CHOL clusters in the $\mathrm{L}_{\mathrm{o}}$ phase. These clusters are reminiscent of those found in atomistic simulations of cholesterol analogs, ${ }^{27}$ but as we will show below their formation is more strongly favoured in the CG models and they can be inhibited by increasing the sterol size.

The article is organized as follows. Firstly, we report details of the molecular dynamics simulation method employed in this work, the lipid forcefields and the analysis tools to identify and quantify the clusters. We then report our main results for the lateral sterol structure as a function of sterol size as well as the impact of the sterol type on the gel/ $\mathrm{L}_{\mathrm{d}}$ and gel/ $/ \mathrm{L}_{\mathrm{o}}$ phase transitions. A final section with our conclusions and a final discussion on the relevance of our results to the superlattice and umbrella models closes the paper.

\section{Methods}

\subsection{Forcefield and simulation details}

The MARTINI 2.0 26 coarse-grained forcefield was employed in all our computations. This forcefield has been tested extensively in computer simulations of biomolecular systems, and we have recently used it to compute the phase diagram of DPPC as a function of cholesterol concentration, $\frac{14}{14}$ hence it represents a good starting point for our investigation.

The molecular dynamics simulations were performed us- ing bilayer membranes of fully saturated 1,2-dipalmitoylsn-glycero-3-phophocholine (DPPC) containing either 20 or $50 \mathrm{~mol} \%$ of cholesterol (CHOL). The DPPC and CHOL molecules were represented using the MARTINI approach, coarse-graining the atomic representation into a molecular one consisting of beads, where each bead represents typically 4 atoms, except for cholesterol which is represented by 8 pseudoatoms. Water was modelled using the original MARTINI parameters. Given the temperature range considered in our simulations it was not necessary to add antifreeze particles. In addition to the original MARTINI CHOL molecule, we simulated two additional sterol molecules, henceforth called CHOLL and CHOLS. In these sterols the diameter of all the CHOL beads was increased (CHOLL) or reduced (CHOLS) by a factor of 1.15 and 0.85 respectively. As we will see below this size change significantly influences the membrane phase behaviour and cholesterol structure in the bilayer, in line with the experimental behaviour observed in naturally occurring sterols. The interaction strength was the same as that employed in the original MARTINI parameters for CHOL. The interactions between sterol-sterol, sterol-DPPC and DPPCDPPC molecules were computed using the standard combining rules.
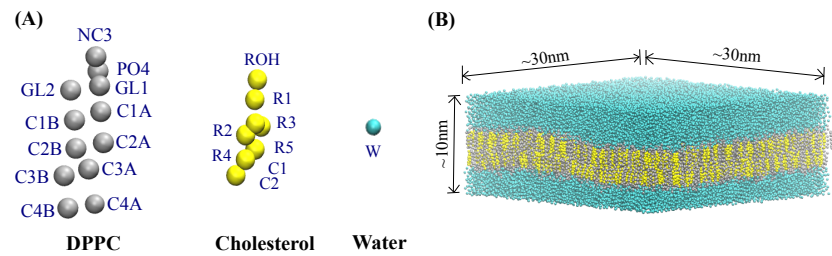

Fig. 1 (A) Coarsed-grained DPPC, cholesterol (CHOL) and sterols (CHOLL and CHOLS), and water models employed in this work. (B) Snapshot of a DPPC bilayer with a (50:50)\% (DPPC:CHOL) composition.

The membranes consisted of either 2432 DPPC lipids and 640 sterols, or 2304 DPPC lipids and 2304 sterols, to achieve the desired $20 \%$ and $50 \%$ compositions, respectively. In order to simulate fully hydrated bilayers, we added between 38000 and $50000 \mathrm{CG}$ water beads. This level of hydration is equivalent to $\sim 50$ "real" water molecules per lipid.

The simulations were performed using the GROMACS package (vs. 4.5) ${ }^{28}$ at constant pressure, zero surface tension and constant temperature. The semi-isotropic Berendsen barostat ${ }^{29}$ was applied separately in the $x / y$ and $z$ directions. The pressure in the direction normal to the bilayer plane was set to 1 bar with a barostat time constant of 2 ps. The lipid bilayer and water were maintained at constant temperature separately by coupling them to separate Berendsen thermostats, using a time constant of 2 ps. Periodic boundary conditions were applied in all three spatial directions. The equations of motion 
were integrated using the leap-frog algorithm ${ }^{30}$ with a time step of $20 \mathrm{fs}$. Each bilayer was simulated for $\sim 2 \mu \mathrm{s}$. The first $\mu$ s was discarded to ensure the averages were computed using well equilibrated configurations.

The non-bonded interactions were modelled using the shifted Lennard-Jones (LJ) 12-6 potential, with the forces decaying smoothly to zero between 0.9 and $1.2 \mathrm{~nm}$, while $1.2 \mathrm{~nm}$ was adopted as cutoff for both the LJ and Coulombic interactions. The coulombic interactions were handled by using a shifted coulombic potential throughout the whole distance interval, 0-1.2 nm, combined with a relative dielectric constant of 15 .

To investigate the influence of the modified sterols on the membrane phase behaviour we performed simulations in the temperature range, $283-333 \mathrm{~K}$. The main gel- $\mathrm{L}_{\mathrm{d}}$ transition temperature for the DPPC MARTINI model is in this temperature interval.

\subsection{Analysis}

We have employed several order parameters to quantify the impact of the sterol molecules on the lateral bilayer structure and phase behaviour. The area per lipid was determined using the Voronoi tessellation method. 31 The Voronoi method provides a powerful approach to investigate the geometric structure of liquids ${ }^{32}$ and to analyse the lateral organisation of the bilayer structure. 14 The construction was performed by projecting the coordinates of the PO4 beads of the DPPC lipids and the $\mathrm{ROH}$ beads of the CHOL, on to the bilayer plane (xy) (See Figure 1A for bead labels). The Voronoi polygons were then constructed and assigned to either DPPC or CHOL molecules. The areas of these polygons were averaged to extract the area per lipid and area per cholesterol. As the bilayer is isotropic in composition we performed an average over both leaflets in order to increase the statistical accuracy of our calculations. The computations were performed with the Qhull library. $\underline{33}$

The 2D radial distribution functions (RDF) were computed to quantify the long range lateral structure in the bilayer plane. We used the coordinates of two beads in the middle of the DPPC acyl chains (C2A and C2B, see figure 1), hence following a similar approach to that used in atomistic models. 34 These beads are immersed in the hydrophobic region and provide a clear view of the lateral packing of the phospholipids.

The lipid diffusion coefficients were obtained from the analysis of the mean-square displacement (MSD), $\left\langle\Delta r(t)^{2}\right\rangle \sim t^{\alpha}$, where $t$ is the time. In a typical diffusive process the exponent $\alpha$ should be equal to 1 but sub-diffusive behaviour, $\alpha=1 / 2$ is not uncommon in the crowded environment of biological membranes. Indeed, sub-diffusion $(\alpha<1)$ has been reported at timescales reaching $\sim 10^{-1} \mu \mathrm{s}$ in simulations of DPPC/CHOL mixtures ${ }^{14 \sqrt[35]{3}}$ and at longer timescales for pro- teins in bilayers. ${ }^{36}$ We ensured that the MSD diffusive regime had been reached before calculating the diffusion coefficient of the phospholipids, $D_{\text {DPPC }}$. The latter was obtained from the Einstein relation in $2 \mathrm{D}$ :

$$
\begin{aligned}
\left\langle\Delta r(t)^{2}\right\rangle & =\frac{1}{N_{D P P C}} \sum_{i=1}^{N_{D P P C}}\left\{\mathbf{r}_{i}(t)-\mathbf{r}_{i}(0)\right\}^{2} \\
D_{\mathrm{DPPC}} & =\lim _{t \rightarrow \infty} \frac{1}{4 t}\left\langle\Delta r(t)^{2}\right\rangle
\end{aligned}
$$

where the brackets \langle\rangle denote a time average. We used $2 \times$ $10^{4}$ configurations evenly distributed over a $2 \mu$ s trajectory to quantify the lipid diffusion. The centre of mass motion of the simulation box was removed to eliminate possible errors in the computation of the MSD. 37

The sterol cluster structure was analysed by using the Voronoi construction. For a given configuration and for each bilayer leaflet we projected on the bilayer plane $(x y)$ the coordinates of the R3 bead in the sterol molecule and the $\mathrm{C} 2$ beads in the DPPC lipids (see figure 1). Two sterols were considered to be part of the same cluster if their Voronoi polygons shared at least one edge. Additional Voronoi polygons (molecules) fulfilling this conditions were added to a given cluster. The cluster construction was automatised using a Python script. Once all the clusters were constructed we quantified their shape by computing the radius of gyration tensor $\mathbf{S} .38[39 \mathrm{We}$ consider a cluster on the $x, y$ plane, consisting of $n_{s}$ atoms with the cluster center of mass defined such that $\sum_{i=1}^{n_{s}} s_{i}=0$, where $s_{i}=\operatorname{col}\left(x_{i}, y_{i}\right)$ are the coordinates of atom $i$ in the cluster. The radius of gyration can be obtained from,

$$
\mathbf{S}=\frac{1}{n_{s}} \sum_{i=1}^{n_{s}} s_{i} s_{i}^{T}=\left(\begin{array}{cc}
S_{x x} & S_{x y} \\
S_{y x} & S_{y y}
\end{array}\right)
$$

Note that we computed the 2D tensor using the coordinates, $x$ and $y$, defining the bilayer plane. The asphericity of the $d$ dimensional cluster, $A_{d}$, is given by 40

$$
A_{d}=\frac{1}{d(d-1)} \frac{\operatorname{Tr}\left(\Delta^{2}\right)}{\lambda^{2}}
$$

where $\lambda$ is the average of the eigenvalues obtained from the diagonalization of the radius of gyration tensor, and $\Delta=\mathbf{S}-$ $\lambda \mathbf{I}$, with $\mathbf{I}$ being the identity matrix. The cluster asphericity ranges from $A_{d}=1$, for a perfectly linear cluster, to $A_{d}=0$ for a circular cluster.

The chain order parameter, $S_{\text {chain }}$, was used to measure the conformational order of the DPPC hydrocarbon tails. The order parameter measures the orientation of the lipid tail bond vectors with respect to the bilayer normal and is defined as 14 :

$$
S_{\text {chain }}=\left\langle\frac{1}{2}\left(3 \cos ^{2} \theta-1\right)\right\rangle
$$


where $\theta$ is the angle that the bond vector between two consecutive coarse-grained beads makes with the bilayer normal. The brackets, \langle\rangle , denote an average over tail beads and over time.

\section{Results and discussion}

\subsection{Sterol cluster structure and bilayer phase behaviour at high sterol concentrations}

In our previous work ${ }^{14}$ we found evidence for the formation of linear clusters of cholesterol in DPPC/CHOL bilayers at high CHOL content, $x_{\text {sterol }}=0.5$. We analyze and quantify in the following the average size and shape of the sterol clusters, and evaluate the impact of the sterol size on the cluster structure and bilayer phase behaviour.

We show in figure 2A the C2A-R5 (DPPC-sterol) and R5R5 (sterol-sterol) radial distribution functions (RDFs) projected on the $x y$ bilayer plane at $313 \mathrm{~K}$. The RDFs clearly show the impact of the sterol size. The position of the first peak in the R5-R5 RDF shifts to longer distances as the sterol size increases. We find that the positions of the main peak in the R5R5 RDFs, $\left(r_{\text {main }, \mathrm{CHOLS}} / r_{\text {main }, \mathrm{CHOL}}, r_{\text {main }, \mathrm{CHOLL}} / r_{\text {main }, \mathrm{CHOL}}\right)=$ $(0.82,1.13)$ correlate well with the changes in the effective diameters of the CHOLS and CHOLL sterols with respect to the CHOL one, $(0.85,1.15)$ (see section 2.1). The shape of the R5-R5 first peak features a distinctive double peak in CHOLS and CHOLL, whereas in CHOL shows a single peak, indicating a more localized lateral correlation in the latter case. We find similar shifts in the main peak of the phospholpidcholesterol correlations (see C2A-R5 RDFs in figure 2A). The sterol type also influences the RDF long range order. We find strong long-range oscillations in the RDF structure for the small sterol, CHOLS. Advancing the discussion below, we find that this RDF structure reflects the better packing properties of the CHOLS sterol with the DPPC aliphatic chains, which ultimately stabilizes the gel phase at high temperatures. We show in figure $2 \mathrm{~B}$ the dependence of the area of DPPC and cholesterol $\left(x_{\text {sterol }}=0.5\right)$ on the scaling factor $f$, employed to model the CHOLL and CHOLS molecules. We have considered two temperatures, below and above the main melting phase transition $\left(\mathrm{gel} / \mathrm{L}_{\mathrm{d}}\right.$ ) of the MARTINI DPPC model. The area per sterol increases monotonically with the scale factor (sterol size). We find that the three sterols have a different condensing efficiency (reduction in the area per lipid). The smallest sterol induces a reduction in the area per DPPC with respect to cholesterol (CHOL), while the largest sterol (CHOLL) has a minor impact on the area per DPPC. These different trends reflect the different packing abilities of the sterols, and result in different mesoscopic structures (clustering). Generally, the results discussed above depend very little on temperature, in the interval $283-313 \mathrm{~K}$.

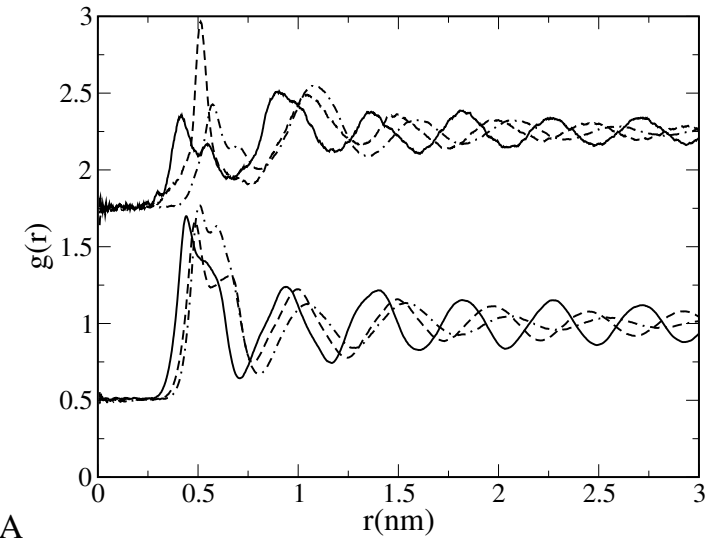

A
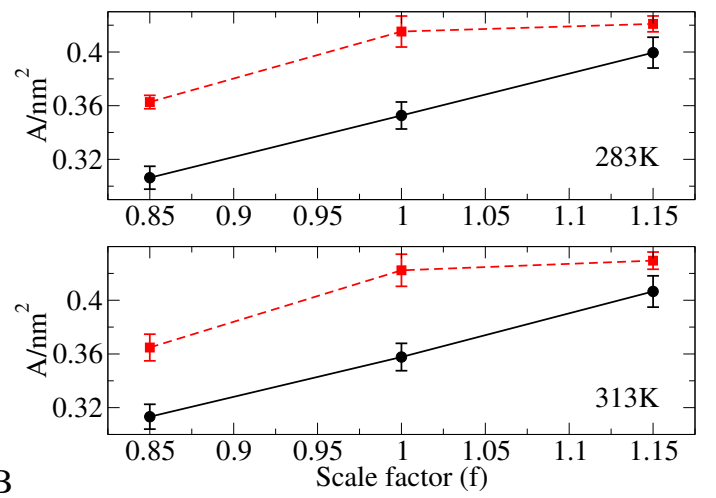

B

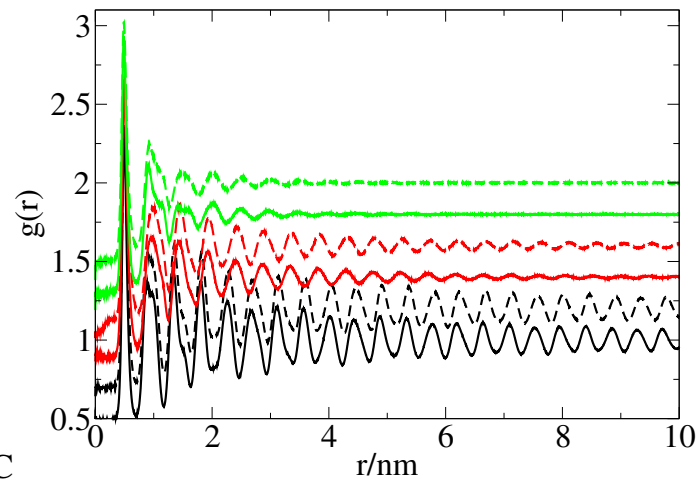

Fig. 2 (A) Radial distribution function C2A-R5 (bottom) and R5-R5 (top - shifted vertically 1.25 units) for the different sterols investigated in this work at $313 \mathrm{~K}$ and $x_{\text {sterol }}=0.5$. CHOLS - full lines; CHOL - dashed lines; CHOLL - dot-dashed line. (B) Dependence of the DPPC (red solid square) and cholesterol (black solid circle) areas with the sterol scale factor $f$ at $283 \mathrm{~K}$ (top) and $313 \mathrm{~K}$ (bottom). The area per lipid was obtained from the Voronoi analysis discussed in section 2.2. (C) Two dimensional radial distribution functions of DPPC in DPPC/sterol bilayers. The RDFs were computed using the positions of the DPPC central beads, C2A and C2B. Full and dashed lines correspond to $283 \mathrm{~K}$ and $313 \mathrm{~K}$, respectively. DPPC/CHOL (middle two RDFs in red), DPPC/CHOLS (bottom two in black) and DPPC/CHOLL (top two in green). 
The changes in molecular area induced by the different sterols can be understood by examining the DPPC lipids translational order, which can be quantified by computing the two dimensional radial distribution function (see figure $2 \mathrm{C}$ ). The RDFs show a distinctive behaviour for the three sterols. The CHOL RDF features a long range decay that we associated in our previous work with the formation of an $\mathrm{L}_{\mathrm{o}}$ phase. ${ }^{14} \mathrm{In}-$ deed according to the phase diagram proposed in that work, the thermodynamic states at $283 \mathrm{~K}$ and $313 \mathrm{~K}$ and $x_{\text {sterol }}=0.5$ lie within the $\mathrm{L}_{0}$ phase region. The DPPC RDF for the small sterol, CHOLS, shows a strong long range order, which is reminiscent of the one observed in a gel phase. In contrast we find that the larger sterol, CHOLL, considerably disrupts the bilayer 2D structure. In this case the lateral RDF features a short range order that is similar to that observed in the $\mathrm{L}_{\mathrm{d}}$ phase. To better understand the modifications of the bilayer structure with changes in sterol size, we computed the DPPC chain order parameter and diffusion coefficients. In all cases the order parameter is high, $S_{\text {chain }} \approx 0.8$, a value which is compatible with a gel or $\mathrm{L}_{\mathrm{o}}$ phase, indicating a large degree of order along the chain. The diffusion coefficient of DPPC in the CHOLL case at $313 \mathrm{~K}$ is $\sim 9 \times 10^{-8} \mathrm{~cm}^{2} / \mathrm{s}$, of the order of the diffusion expected for the fluid phase in this MARTINI model. 14

The cluster analysis reveals distinctive and important modifications in the bilayer lateral structure. We show in figure 3 typical snapshots of one of the monolayers in a DPPC/sterol bilayer. The snapshots were constructed by assigning each Voronoi polyhedron to a DPPC or sterol lipid. We have highlighted the "sterols" belonging to the same cluster by representing their corresponding polyhedra in the same colour. The colour code gives information on their asphericity. The clustering analysis reveals notable changes in the bilayer structure. Small (CHOLS) and medium (CHOL) size sterols form 1D-clusters mainly, with evident chain like structures (see figure 3). In contrast larger sterols (CHOLL) disrupt considerably the formation of chain-like clusters, and we observe a large preference to form 2D disc-like clusters. This general trend is fairly independent of the temperature, visible at 283 and $313 \mathrm{~K}$ (see figure 3). The Voronoi construction also provides information on the sterol-phospholipid packing. Small and medium size sterols (CHOLS, CHOL) pack better in the phospholipid bilayer preserving the DPPC hexagonal structure, while the large sterol, CHOLL, considerably disrupts the long range order. This observation is consistent with the general trends discussed in the analysis of the RDFs (see figure 2).

We have quantified the local structure around a given sterol molecule by performing a Delaunay triangulation analysis. ${ }^{41}$ For this analysis we projected the coordinates of the PO4 DPPC bead and the ROH sterol bead on the $x y$ plane. We show in figure 4 the normalized probability distribution, $P$, of finding a particular lipid (DPPC or sterol) as the nearest neighbour of a given sterol. We find, irrespective of the sterol type,
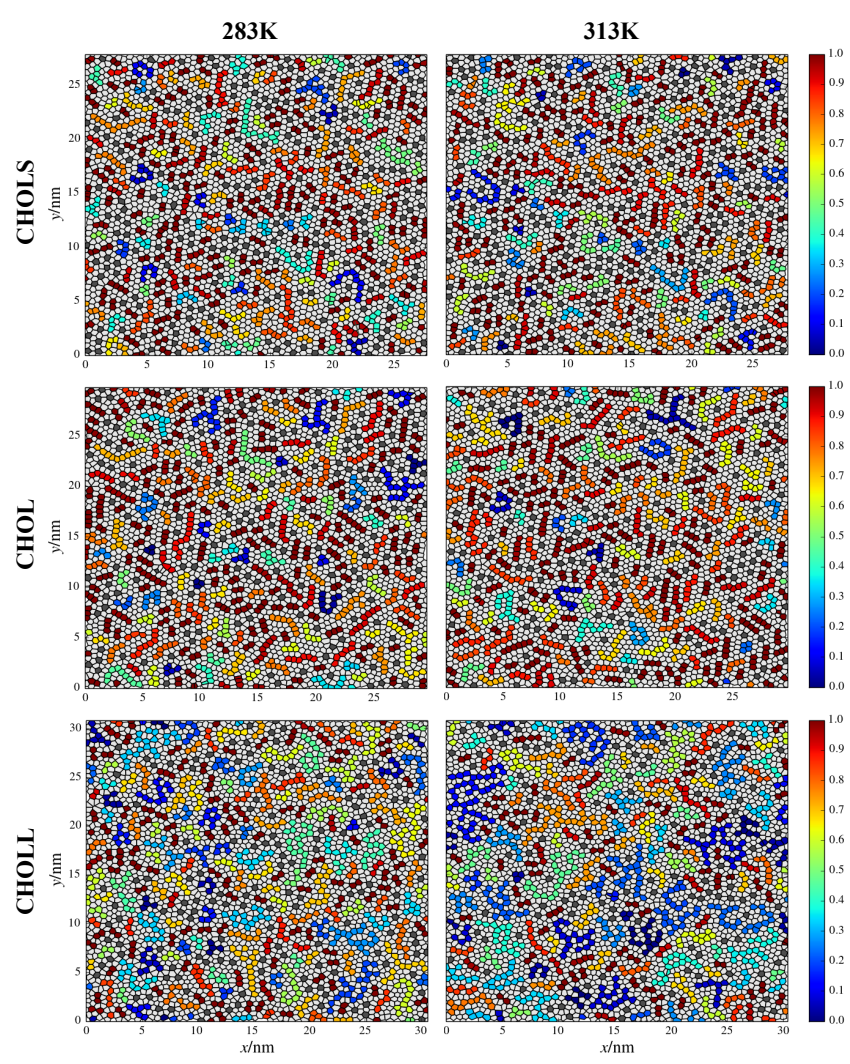

Fig. 3 Snapshots of representative bilayer leaflets showing the Voronoi construction at $283 \mathrm{~K}$ (left) and $313 \mathrm{~K}$ (right). Results for the different sterol molecules, CHOLS (top two panels), CHOL (middle panels) and CHOLL (bottom panels) are shown. Light grey polygons correspond to DPPC lipids and dark grey polygons correspond to single sterols not part of a cluster. The remaining polygons represent sterols which are part of a cluster and these are colour coded according to the asphericity of their cluster, calculated from equation 4

that it is more likely to find a DPPC lipid next to a sterol one, showing that sterol-sterol interactions are less favourable than sterol-lipid ones, as predicted by the umbrella and superlattice models. On average each sterol is surrounded by 2 sterol and 4 DPPC molecules. Even within the disc-shaped clusters formed by CHOLL, each CHOLL molecule tends to associate on average with just two other CHOLL molecules. This indicates that the disc-shaped clusters are not enriched in sterol molecules and that the preference for forming linear or circular clusters is due to the way in which the sterol neighbours are arranged and pack around each other, rather than formation of regions of heterogeneous composition. The average number of neighbours around a point in a Delaunay triangulation is 6 due to Euler's theorem. ${ }^{[2]}$ However, in total each sterol is surrounded by 5 or 6 nearest neighbours making the 
average coordination slightly less than 6 . At $313 \mathrm{~K}$ we obtained, 5.76, 5.66 ad 5.87 for CHOLS, CHOL and CHOLL, respectively. These results reflect the smaller cross-sectional area of a sterol molecules compared to DPPC and it is compensated for by DPPC molecules having an average of slightly more than 6 neighbours.
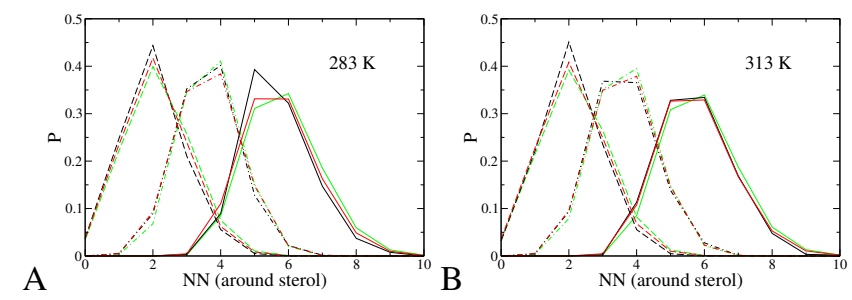

Fig. 4 Normalized probability distribution for finding $N N$ nearest neighbours around a given sterol molecule; CHOLS (red), CHOL (black) or CHOLL (green), at $x_{\text {sterol }}=0.5$. The nearest neighbours are represented as follows: sterol - dashed lines, DPPC -

dash-dotted lines and all lipids (sterols and DPPC molecules) - solid lines. Panels A and B show results for $283 \mathrm{~K}$ and $313 \mathrm{~K}$, respectively.

To demonstrate that the difference between linear and disc shaped clustering with different sized sterols is significant, we have compared our simulation results to a random system. For the random system, we use a $2 \mathrm{D}$ box of the same area and filled with the same number of molecules as in our simulations, with $x_{\text {sterol }}=0.5$ (see figure 5). The molecules are represented as hard, circular discs, with each CHOL molecule represented by a single disc and each DPPC molecule represented by a dimer of discs to account for the two hydrocarbon tails (the $\mathrm{C} 2 \mathrm{~A}$ and $\mathrm{C} 2 \mathrm{~B}$ beads used in the Voronoi tessellation (figure 3). The CHOL and DPPC disc sizes were chosen such that the area per DPPC and per CHOL in the random system, obtained from the Voronoi construction, closely matched the values we calculate from our simulations. The use of this
A

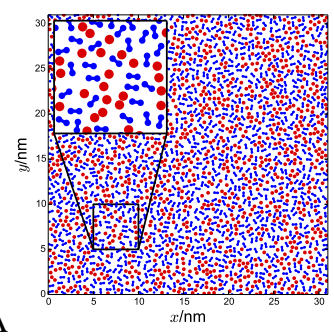

B

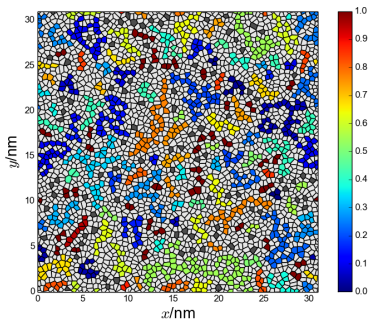

Fig. 5 (A) Typical random system configuration, with $\mathrm{CHOL}$ in red and DPPC in blue. The inset shows a close up part of the system so that the random packing and dimer shape of the DPPC molecule can be seen. (B) Voronoi tesellation of configuration shown in (A), the colouring is exactly the same as in figure 3 indicating the asphericity of the CHOL clusters. excluded volume in our random system was necessary to produce configurations with more uniform density in the $x y$ plane.

To classify the types of clusters formed, we plot cluster size against asphericity and compare the regions of the plots which are highly populated (see figure 6 and note that the colouring to represent number of clusters is plotted on a logarithmic scale). For the small and medium sized sterols (CHOLS and CHOL), the heat maps show a larger density of points towards the top left hand corner of the plot, indicating a larger number of relatively small, linear clusters (asphericities close to 1). However, for the larger sterol (CHOLL) the colour distribution is more even along the asphericity axis, showing that CHOLL has less of a preference for linear clusters, with a larger number of more disc-shaped, low asphericity clusters present. The random system most closely resembles the CHOLL simulations, with clusters distributed fairly evenly over the full range of possible asphericity values. Cluster size also increases with sterol size, from a narrow size range in CHOLS, to wider distributions for $\mathrm{CHOL}$ and again for CHOLL, especially at low asphericities due to the higher multiplicity of these structures. The random system produces a much larger range of cluster sizes, highlighting the importance of the relatively unfavourable sterol-sterol interactions in limiting cluster size in simulations. The comparison with a random system clearly reinforces the observation that the lateral organisation of small and medium sized sterols is non-random and strongly favours the formation of linear clusters. However, a small increase in sterol size to CHOLL is sufficient to remove this preference and disrupt the linear organisation in favour of more rounded, disc-like clusters.

So far, we have shown that the sterol size has a significant impact on the lateral structure of the bilayer. We focus in the following on the influence of the sterol size on the membrane thickness. We show in figure 7 , the number density profiles for the $\mathrm{PO}_{4}$ and $\mathrm{ROH}$ beads in the DPPC and sterol lipids, respectively, at $313 \mathrm{~K}$. The bilayer thickness was estimated from the $\mathrm{PO}_{4}-\mathrm{PO}_{4}$ distance. Swapping CHOL with the largest sterol CHOLL has a negligible impact on the bilayer thickness.

To resolve the location of the sterol groups in the bilayer we have calculated the distance, $d$, between the $\mathrm{ROH}$ and $\mathrm{PO}_{4}$ beads (similar results are obtained if the distance is computed considering the DPPC and sterol center of mass positions instead). For all the three sterols the ROH bead protrudes less towards the water phase, and sits below the $\mathrm{PO}_{4}$ bead. Our results indicate that the relative height of the sterol with respect to DPPC, as measured by $d$, is the same for all three sterols $(\sim 0.52 \mathrm{~nm})$, within the uncertainty of the computations. This shows that the relative position of the sterol with respect to DPPC is not influenced by the sterol size or bilayer phase. Note that CHOLS stabilizes a gel phase while CHOL stabilizes the $\mathrm{L}_{\mathrm{o}}$ phase.

Finally, we have calculated the probability distribution for 

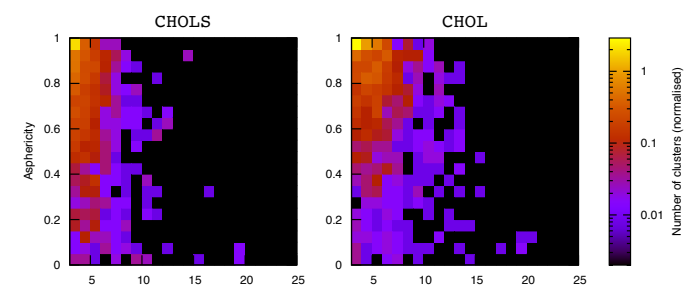

CHOLL
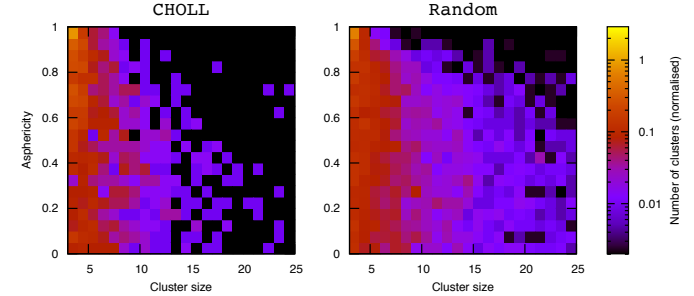

Fig. 6 Heat maps showing the probability of finding clusters of a specific size and asphericity at $313 \mathrm{~K}$ for the different sterol molecules, CHOLS (top left), CHOL (top right), CHOLL (bottom left $)$ and also for a random DPPC/CHOL $\left(x_{\text {sterol }}=0.5\right)$ system (bottom right). The distributions have been normalized considering all the clusters observed in the computations. We have represented in the graphs clusters larger than 2 .

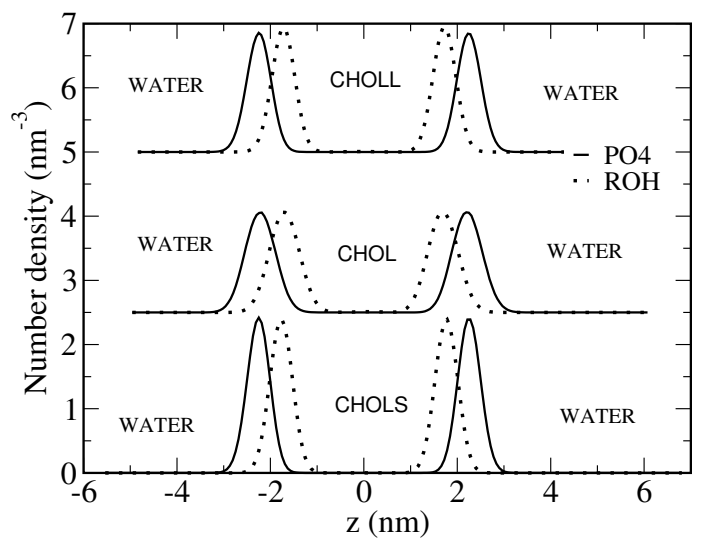

Fig. 7 Number density of the PO4 (DPPC, full line) and ROH (sterol, dashed line) pseudoatoms at $313 \mathrm{~K}$. The results for the CHOL and CHOLL sterols have been shifted upwards 2.5 and 5 units respectively.

the sterol tilt angle (see figure 8) defined as the angle between the vector connecting the R1-R5 beads in the sterol (see figure 1) and the vector normal to the bilayer plane. We find that the angle distribution is insensitive to the sterol size and the average tilt angle of $9-10^{\circ}\left(11^{\circ}\right.$ for CHOLL at $\left.313 \mathrm{~K}\right)$ agrees well with previous atomistic simulation studies ${ }^{43}$ and is close to the experimental value of $16^{\circ}$ for a DPPC/CHOL $\left(x_{\mathrm{CHOL}}=0.5\right)$ bilayer at $303 \mathrm{~K}^{44}$. However, the increase in tilt angle which occurs in the gel phase is not reproduced here in the low temperature, small sterol simulations, reflecting a known limita- tion of the MARTINI model. ${ }^{45}$ In figure $8 \mathrm{~B}$, we compare the tilt angle distributions from simulations to the probability distribution for the orientation of a system of ideal rods along a given direction, given by $P_{0}=\sin (\theta)$. In this system the probability distribution is determined solely by the multiplicity of states with a given tilt angle and so by plotting $P(\theta) / P_{0}$, the entropic contributions to the tilt angle can be removed. Figure $8 \mathrm{~B}$ shows that all sterols show a strong preference to align parallel to the bilayer normal, although this preference is weakest for CHOLL. This, along with the slightly broader tilt angle distribution for CHOLL (see figure $8 \mathrm{~A}$ ), reflects the more fluid, less ordered conformation of the CHOLL bilayer.

A
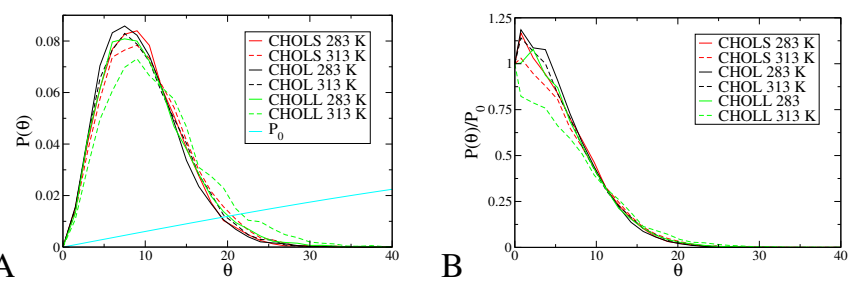

Fig. 8 (A) Normalised probability distribution, $P(\theta)$, for the angle between the vector normal to the bilayer plane and the vector connecting beads R1 - R5, for bilayers with $x_{\text {sterol }}=0.5$. (B) $P(\theta) / P_{0}$ derived from panel (A). The colouring in both graphs is CHOLS - red, CHOL - black and CHOLL - green, the solid lines are simulations at $283 \mathrm{~K}$ and the dashed lines are at $313 \mathrm{~K}$.

\subsection{Phase behaviour at low cholesterol content, $x_{\text {sterol }}=$ 0.2}

In this section we investigate the impact of sterol size on the DPPC/sterol bilayer main phase transition, i.e. gel $/ \mathrm{L}_{\mathrm{d}}$. We found in our previous work ${ }^{144}$ that this transition is still present at a cholesterol composition of $x_{\text {sterol }}=0.2$, with a melting temperature of $\sim 310 \mathrm{~K}$. We generated sterol/DPPC configurations by replacing the original CHOL molecules by CHOLS and CHOLL. These configurations were then equilibrated over $\mu s$ timescales, before performing structural and dynamic analyses.

We find that the area per lipid and area per sterol depends strongly on the sterol type (see figure $9 \mathrm{~A}$ ). For the original cholesterol molecule, CHOL, we observe a discontinuous change in the area, between $303 \mathrm{~K}$ and $313 \mathrm{~K}$, which is connected to the $\mathrm{gel} / \mathrm{L}_{\mathrm{d}}$ first order transition discussed in previous work. ${ }^{14}$ However, for small CHOLS molecules, we do not find evidence for the transition in this temperature interval. Both, the area per DPPC and per CHOLS are low, with values typical of a gel phase, hence indicating that the small sterol stabilizes the gel phase shifting the melting transition to higher temperatures. The stabilization of the gel phase can be confirmed by analysing the lateral radial 


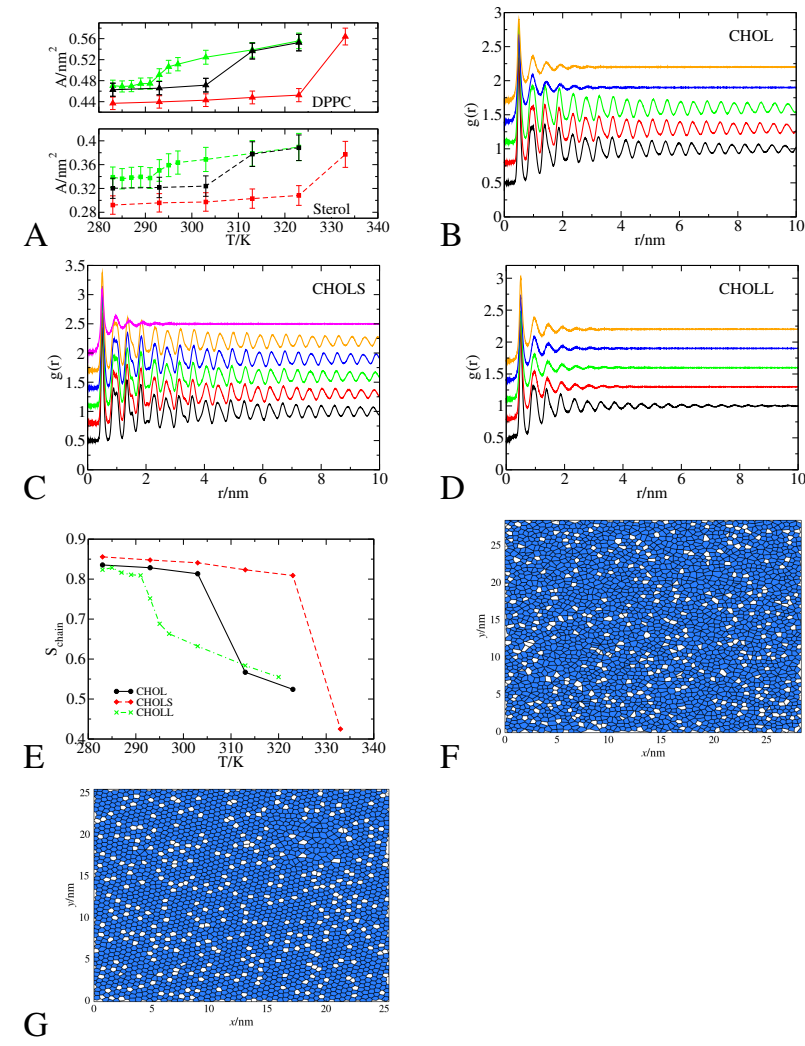

Fig. 9 (A) Area per DPPC (solid lines) and sterol (dashed lines) obtained from the Voronoi construction in DPPC/CHOL (black), DPPC/CHOLL (green) and DPPC/CHOLS (red) bilayers with $x_{\text {sterol }}=0.2$. (B, C and D) 2D RDFs of DPPC-DPPC pair correlation computed using $\mathrm{C} 2 \mathrm{~A}$ and $\mathrm{C} 2 \mathrm{~B}$ beads at various temperatures (from bottom to top: $283 \mathrm{~K}, 293 \mathrm{~K}, 303 \mathrm{~K}, 313 \mathrm{~K}, 323 \mathrm{~K}$ and $333 \mathrm{~K}$ for the CHOLS sterol) in DPPC/CHOL (B), DPPC/CHOLS (C) and DPPC/CHOLL (D) bilayers. RDFs are shifted vertically by 0.3 units for clarity. (E) $S_{\text {chain }}$ obtained by averaging over all acyl beads. (F) and $(\mathrm{G})$ : Voronoi construction of representative bilayer leaflets in the presence of CHOLS at $333 \mathrm{~K}(\mathrm{~F})$ and $323 \mathrm{~K}(\mathrm{G})$. Voronoi polygons for DPPC are coloured in blue, and polygons for CHOLS in white.

distribution functions (RDF) (see figures $9 \mathrm{~B}, \mathrm{C}$ ). For the CHOL molecules we observe behaviour consistent with the gel $/ L_{d}$ phase transition. The radial distribution function looses the long range oscillatory behaviour at a specific temperature range, which agrees with the discontinuous change in area per lipid reported in figure $9 \mathrm{~A}$. The RDFs of the bilayers containing small sterols, CHOLS, feature long range oscillatory behaviour up to $323 \mathrm{~K}$, which is consistent with the existence of a gel phase at that temperature. At higher temperatures, 333 $\mathrm{K}$, the lateral DPPC RDF shows the structure characteristic of the liquid disordered phase, i.e. short range oscillations, with no indication of lateral ordering beyond $3 \mathrm{~nm}$ (see figure $9 \mathrm{C}$ ). Snapshots of representative monolayers in the CHOLS bilayer confirm the existence of the gel $/ \mathrm{L}_{\mathrm{d}}$ transition in the interval 323-333 K (see figures 9F, G). This transition can be further confirmed by analyzing other order parameters. We find that the area per DPPC features a discontinuous jump between 323 and $333 \mathrm{~K}$, reaching $0.564 \pm 0.016 \mathrm{~nm}^{2}$ at $333 \mathrm{~K}$, a value typical of the $\mathrm{L}_{\mathrm{d}}$ phase. The orientational order parameter at this temperature, $S_{\text {chain }}=0.423$, is also characteristic of a fluid phase. Overall, our results strongly support the notion that small sterol molecules stabilize the gel phase, shifting the melting temperature of the gel/ $\mathrm{L}_{\mathrm{d}}$ transition (allowing for hysteresis effects) to $\sim 328 \mathrm{~K}$.
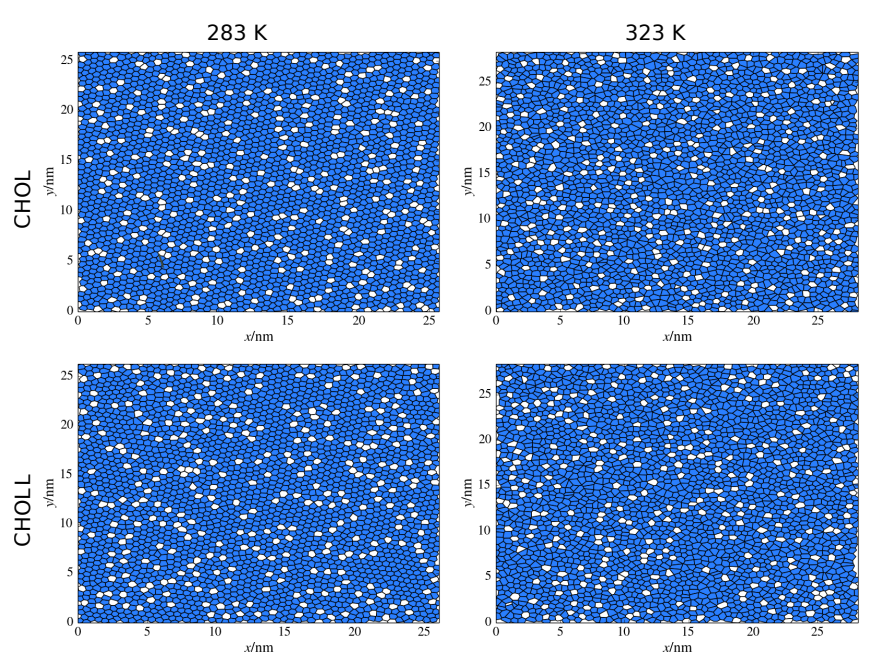

Fig. 10 Voronoi construction of one monolayer for a DPPC/CHOL bilayer $\left(x_{\text {sterol }}=0.2\right)$ at $283 \mathrm{~K}$ (top-left) and $323 \mathrm{~K}$ (top-right); for DPPC/CHOLL at $283 \mathrm{~K}$ (bottom-left) and $323 \mathrm{~K}$ (bottom-right). DPPC is represented in blue and the sterols in white.

The larger sterol, CHOLL, has the opposite effect on the position of the phase transition, causing it to move to lower temperatures. This is indicated by the position of the discontinuous jump in both the area per lipid and chain order parameter over the temperature range of 289 - $297 \mathrm{~K}$ (see figures 9A, E). However, CHOLL also has a significant effect on the structures of the two phases involved in the transition. In CHOL and CHOLS, the low temperature phase is a gel, which can be confirmed by noting the long range correlations in the RDFs at low temperatures (see figures $9 \mathrm{~B}, \mathrm{C}$ ) indicating a regular arrangement of molecules, in this case on a hexagonal lattice. However the CHOLL RDF (see figure 9D) shows a structure intermediate between that of the other sterols at low and high temperatures. This phase represents a disordered gel phase, in which the larger CHOLL molecule no longer fits neatly into the underlying hexagonal lattice, formed by the lipid tails in the gel. Instead the CHOLL acts as an impurity, distorting the underlying lattice and so reducing the long range periodicity. 
An example of this structure is shown in figure 10 (bottom left panel) and can be compared with the CHOL gel structure figure 10 (top left panel). In both cases, the hexagonal packing of DPPC molecules is clearly visible, however, around CHOL molecules the hexagonal packing is much better preserved than around CHOLL molecules.

On the high temperature side of the CHOLL transition is a fluid phase, which is confirmed by the diffusion coefficients (see figure 11 and table 1). However, the appearance of this phase at a lower temperature than usual, coupled with the significant temperature dependence of $S_{\text {chain }}$ in the fluid phase (note the high rate of change of $S_{\text {chain }}$ with temperature in figure $9 \mathrm{E}$ ) means that it is considerably more ordered than the $\mathrm{L}_{\mathrm{d}}$ phase formed in CHOL simulations. Whilst the change in chain order during the transition is reduced with the larger sterol, the diffusion coefficients are less affected, and change by two orders of magnitude over the transition temperature range, from $D_{\text {DPPC }} \sim 10^{-9} \mathrm{~cm}^{2} \mathrm{~s}^{-1}$ typical of the gel phase to $D_{\text {DPPC }} \sim 10^{-7} \mathrm{~cm}^{2} \mathrm{~s}^{-1}$ typical of the fluid phase. These structural and dynamic properties suggest that the phase that forms initially has some characteristics of an $\mathrm{L}_{\mathrm{o}}$ phase, but then becomes more $\mathrm{L}_{\mathrm{d}}$ like as the temperature is increased. Therefore, by increasing the size of the sterol molecule, the position of the phase transition not only shifts to a lower temperature, but the characteristics of the two phases either side of the transition become closer; the gel phase becomes more disordered and the fluid has a higher chain order and smaller area per lipid than a normal $\mathrm{L}_{\mathrm{d}}$ phase.

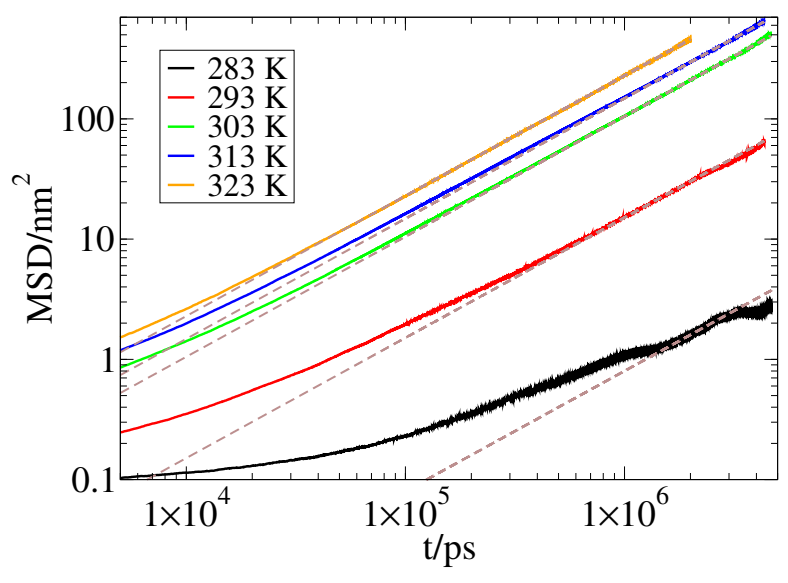

Fig. 11 Mean square displacement (MSD) of DPPC in DPPC/CHOLL bilayers $\left(x_{\text {sterol }}=0.2\right)$ as a function of temperature. Dashed lines represent the expected behaviour of the MSD in the diffusive regime.
Table 1 Lateral diffusion coefficient of DPPC, $D_{\text {DPPC }} / 10^{-7} \mathrm{~cm}^{2} \mathrm{~s}^{-1}$ in $x_{\text {sterol }}=0.2$ bilayers. The hyphen "-" represents a system for which diffusive behaviour was not reached in our simulation time scale.

\begin{tabular}{ccc}
\hline \hline T/K & DPPC/CHOL & DPPC/CHOLL \\
\hline 283 & - & 0.02 \\
293 & 0.02 & 0.38 \\
303 & 0.03 & 2.63 \\
313 & 3.79 & 3.70 \\
323 & 5.48 & 5.73 \\
\hline
\end{tabular}

\section{Discussion and Conclusions}

We have examined the role of sterol size on the lateral structure and phase behaviour of DPPC membranes using molecular dynamics simulations and the MARTINI coarse-grained forcefield. Several order parameters were computed to quantify the dependence of lateral order and membrane phase behaviour on sterol type at sterol concentrations of 20 and $50 \mathrm{~mol} \%$.

The sterol size has a large impact on the lateral structure of the bilayer. At high cholesterol concentrations $\left(x_{\text {sterol }}=0.5\right)$, typical of those stabilizing the $\mathrm{L}_{\mathrm{o}}$ phase, the MARTINI model predicts the formation of linear CHOL clusters. Given that the MARTINI CHOL forcefield captures the main qualitative behaviour of cholesterol/DPPC phase coexistence, we expect that the predicted linear clusters should be a general characteristic of sterol lipids. Our results show that the formation of these clusters is fairly sensitive to the sterol size. This sensitivity is reminiscent of the one observed with atomistic models, which suggests that linear clusters are formed when the off plane methyl groups of CHOL are removed.

The smaller sterol, CHOLS also forms linear clusters and has an enhanced condensing effect, significantly reducing the area per lipid compared to the CHOL and CHOLL sterols. In contrast the large sterol, CHOLL, favours the formation of disc like clusters. However for all three sterols, the average environment around each sterol is very similar, with on average two sterol and four DPPC nearest neighbours. This indicates that even at high sterol concentrations $\left(x_{\text {sterol }}=0.5\right)$, sterol molecules prefer to be next to DPPC molecules, as predicted by the umbrella and super lattice models. Interestingly the bilayer thickness is fairly insensitive to sterol size, as is the sterol tilt angle.

We have shown that the bilayer phase behaviour is also strongly influenced by the sterol size. Smaller sterols stabilise the gel phase due to their ability to insert more comfortably into the underlying hexagonal lattice of the gel phase and pack more closely with the DPPC hydrocarbon tails that form this lattice. Reduction of the sterol size leads to a shift ( $\sim 10 \mathrm{~K})$ in 
the melting transition to higher temperatures. Conversely, the larger sterol has the opposite effect, destabilising the gel phase by disrupting and disordering the regular hexagonal packing and long range periodicity. The consequence of this destabilisation of the gel phase, is the formation of a more ordered liquid phase at lower temperatures than can be achieved using the normal CHOL and which has some similarities to an $\mathrm{L}_{\mathrm{o}}$ phase. The small change in molecule size that we introduce is therefore sufficient to partially decouple the translational and configurational changes associated with the $S_{0} / L_{d}$ transition. Slightly larger changes in sterol size could potentially destroy the $\mathrm{S}_{\mathrm{o}} / \mathrm{L}_{\mathrm{d}}$ transition completely to favour a more continuous and gradual transition via a liquid ordered phase.

The dependence of the lateral sterol organisation and phase behaviour on sterol size is due to changes in the packing of the sterol and DPPC molecules within the bilayer. A closer match in size between the sterol and phospholipid aliphatic chains means that the sterol can fit more easily into the underlying, roughly hexagonally coordinated lipid structure with minimal disruption to the bilayer. This in turn favours the formation of linear clusters and stabilises the gel phase. The cross-sectional area of cholesterol is intermediate between that of one and two acyl chains, so small changes in cholesterol size can substantially modify the packing of the sterol and lipids in a bilayer. We note that the changes in the pseudoatom radius involve a relative change with respect to the CHOL molecule of just $15 \%$ for both CHOLL and CHOLS molecules.

One important question is how representative are our CHOLS and CHOLL molecules of real sterols? A comparison can be established with ergosterol and lanosterol. Ergosterol is found in funghi and it has a chemical structure very similar to that of cholesterol, while lanosterol is a precursor to

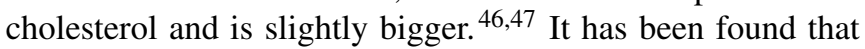
ergosterol promotes the packing of the lipid chains and it has a stronger condensing effect. This is also reflected in the higher melting temperature 48 of the ergosterol/DPPC bilayers (330.5 $\mathrm{K})$. This general behaviour is similar to what we observe for the smaller sterol, CHOLS, which features a similar melting temperature. On the other hand, it has been found in atomistic simulations that lanosterol has a reduced packing and chain ordering ability. ${ }^{46 / 49}$ This behaviour is similar to what we observe with the larger sterol model (CHOLL).

To summarize, we have shown that small changes in sterol size can significantly affect the bilayer phase behaviour and lateral organisation. Our results highlight the importance of the forcefield parameters in modelling the sterol molecules and predicting the correct phase behaviour. The sensitivity of the lateral sterol organisation to sterol size reported here can be relevant to understanding the ability of different sterols to promote raft formation, which is known to correlate with sterol structure.$\underline{50}$ Further, the high sensitivity of the cluster structure to sterol size indicates that experiments performed with cholesterol analogs, e.g. with fluorescent tags, need to be carefully examined to assess whether they reflect the real structure of cholesterol molecules in natural biological membranes.

\section{Acknowledgement}

The authors would like to thank Imperial College High Performance Computing Service for providing computational resources. FB acknowledges EPSRC (EP/J003859/1) for the award of a Leadership Fellowship. We would also like to thank the EPSRC for Programme grant EP/J017566/1 and a PhD Studentship for Imperial College.

\section{References}

1 D. Sadava, D. M. Hillis, H. C. Heller and M. R. Berenbaum, Life: The Science of Biology, Freeman, 2011.

2 R. A. Demel, L. L. M. van Deenen and B. A. Pethica, Biochimica et Biophysica Acta, 1967, 135, 11-19.

3 G. W. Stockton and I. C. Smith, Chemistry and physics of lipids, 1976, 17, 251-63.

4 P. Heftberger, B. Kollmitzer, A. Rieder, H. Amenitsch and G. Pabst, Biophysical Journal, 2015, 108, 854-862.

5 J. Song and R. Waugh, Biophysical Journal, 1993, 64, 1967-1970.

6 D. Brown and E. London, Ann. Rev. of Cell and Developmental Biology, 1998, 14, 111-136.

7 K. Jacobson, O. Mouritsen and G. Anderson, Nature Cell Biology, 2007, 9, 7-14.

8 T. McIntosh, Biophysical J., 2015, 108, 783-784.

9 J. H. Ipsen, G. Karlström, O. Mourtisen, H. Wennerström and M. Zuckermann, Biochimica et Biophysica Acta, 1987, 905, 162 - 172.

10 S. Veatch, O. Soubias, S. Keller and K. Gawrisch, Proc. Nat. Acad. Sci., 2007, 104, 17650-17655.

11 P. Uppamoochikkal, S. Tristram-Nagle and J. Nagle, Langmuir, 2010, 26, 17363-17368.

12 S. Baoukina, E. Mendez-Villluendas and D. Tieleman, J. Am. Chem. Soc., 2012, 134, 17543-17553.

13 W. Drew Bennett and D. Tieleman, Biochimica et Biophysica Acta, 2013, 1828, 1765-1776.

14 Y. Zhang, A. Lervik, J. Seddon and F. Bresme, Chemistry and Physics of Lipids, 2015, 185, 88-98.

15 M. R. Ali, K. H. Cheng and J. Huang, Proceedings of the National Academy of Sciences, 2007, 104, 5372-5377.

16 P. L.-G. Chong, W. Zhu and B. Venegas, Biochimica et Biophysica Acta, 2009, 1788, 2 - 11.

17 A. Radhakrishnan and H. M. McConnell, Biophysical Journal, 1999, 77, 1507-1517.

18 P. L. Chong, Proceedings of the National Academy of Sciences, 1994, 91, 10069-10073.

19 P. J. Somerharju, J. A. Virtanen, K. K. Eklund, P. Vainio and P. K. J. Kinnunen, Biochemistry, 1985, 24, 2773-2781.

20 J. Huang, in Biothermodynamics, Part A, ed. J. M. H. Michael L. Johnson and G. K. Ackers, Academic Press, 2009, vol. 455, pp. 329 - 364.

21 H. Martinez-Seara, T. Róg, M. Pasenkiewicz-Gierula, I. Vattulainen, M. Karttunen and R. Reigada, Biophysical Journal, 2008, 95, 3295 3305.

22 H. Martinez-Seara, T. Róg, M. Karttunen, I. Vattulainen and R. Reigada, The Journal of Physical Chemistry B, 2009, 113, 8347-8356.

23 T. Róg, M. Pasenkiewicz-Gierula, I. Vattulainen and M. Karttunen, Biophysical Journal, 2007, 92, 3346 - 3357. 
24 O. Edholm and J. F. Nagle, Biophysical Journal, 2005, 89, 1827 - 1832.

25 Z. Huang, J. Seddon and R. Templer, Chemistry and Physics of Lipids, 1996, 82, 53-61.

26 S. J. Marrink, H. J. Risselada, S. Yefimov, D. P. Tieleman and A. H. de Vries, The Journal of Physical Chemistry B, 2007, 111, 7812-7824.

27 H. Martinez-Seara, T. Róg, M. Kartunen, I. Vattulainen and R. Reigada, PLOS one, 2010, 5, e11162.

28 B. Hess, C. Kutzner, D. van der Spoel and E. Lindahl, Journal of Chemical Theory and Computation, 2008, 4, 435-447.

29 H. J. C. Berendsen, J. P. M. Postma, W. F. van Gunsteren, A. DiNola and J. R. Haak, The Journal of Chemical Physics, 1984, 81, 3684-3690.

30 R. W. Hockney and J. W. Eastwood, Computer Simulation Using Particles, Taylor \& Francis, 1989.

31 W. Brostow, M. Chybicki, R. Laskowski and J. Rybicki, Physical Review $B, 1998, \mathbf{5 7}, 13448-13458$.

32 J. Montoro, F. Bresme and J. Abascal, Journal of Chemical Physics, 1994, 101, 10892-10898.

33 C. Barber, D. Dobkin and H. Huhdanpaa, ACM Trans. on Mathematical Software, 1996, 22, 469-483.

34 D. Mohammad-Aghaie, E. Mace, C. A. Sennoga, J. M. Seddon and F. Bresme, Journal of Physical Chemistry B, 2010, 114, 1325-1335.

35 J.-H. Jeon, H. M.-S. Monne, M. Javanainen and R. Metzler, Phys. Rev. Lett., 2012, 109, 188103.

36 M. Javanainen, H. Hammaren, L. Monticelli, J.-H. Jeon, M. S. Miettinen, H. Martinez-Seara, R. Metzler and I. Vattulainen, Faraday Discuss., 2013, 161, 397-417.

37 E. Lindahl and O. Edholm, The Journal of Chemical Physics, 2001, 115, 4938-4950.

38 V. Blavatska and W. Janke, The Journal of Chemical Physics, 2010, 133, 184903(1)-184903(7).

39 K. Tay and F. Bresme, Molecular Simulation, 2005, 31, 515-526.

40 W. Gaoyuan, Chinese Journal of Polymer Science (CJPS), 1995, 13, 204.

41 L. Guibas and J. Stolfi, ACM Transations on Graphics, 1985, 4, 74-123.

42 C. S. Smith, A Search for Structure, MIT Press, 1981.

43 A. M. Smondyrev and M. L. Berkowitz, Biophysical Journal, 1999, 77, $2075-2089$

44 R. Murari, M. P. Murari and W. J. Baumann, Biochemistry, 1986, 25, $1062-1067$.

45 S. J. Marrink, J. Risselada and A. E. Mark, Chemistry and Physics of Lipids, 2005, 135, $223-244$.

46 Z. Cournia, G. M. Ullmann and J. C. Smith, The Journal of Physical Chemistry B, 2007, 111, 1786-1801.

47 J. Czub and M. Baginski, Biophysical Journal, 2006, 90, 2368 - 2382.

48 M. Pühse, C. Jeworrek and R. Winter, Chemistry and Physics of Lipids, 2008, 152, $57-63$.

49 D. Huster, H. A. Scheidt, K. Arnold, A. Herrmann and P. Muller, Biophysical Journal, 2005, 88, 1838 - 1844.

50 M. Jiwei Wang and E. London, Biochemistry, 2004, 43, 1010-1018.

This journal is (c) The Royal Society of Chemistry [year]

Journal Name, 2010, [vol], $1-\sqrt{11}$ | 11 